\title{
OPTIMAL DESIGN OF TURBINES WITH AN ATTACHED MASS*
}

\author{
Boris P. Belinskiy ${ }^{1}$, C. Maeve McCarthy ${ }^{2}$ And Terry J. Walters $^{1}$
}

\begin{abstract}
We minimize, with respect to shape, the moment of inertia of a turbine having the given lowest eigenfrequency of the torsional oscillations. The necessary conditions of optimality in conjunction with certain physical parameters admit a unique optimal design.
\end{abstract}

Mathematics Subject Classification. 49K15, 49K30, 34B24, 49R05, 73K10, 73K40.

Received August 30, 2002. Revised December 17, 2002.

\section{INTRODUCTION}

Following the model developed by Collatz [2], we consider the torsional oscillations of a disk $r \in[a, b]$, $0<a<b<\infty$ of variable cross-section attached to a finite cylinder at its middle, see Figure 1 . The cylinder

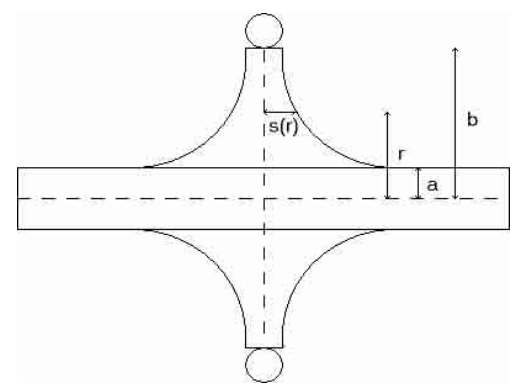

Figure 1. Disk of cross-section $s(r)$ attached to a cylinder.

is fixed at its end points. A mass is attached to the perimeter of the disk. This mechanical system imitates a turbine with a blade and is derived in detail in the Appendix. Our goal is to construct the optimal design of

Keywords and phrases: Optimal design, disk, moment of inertia, Sturm-Liouville problem, least eigenvalue, rearrangement, Helly's principle, Calculus of Variations.

* The first author was supported in part by the University of Tennessee at Chattanooga Center of Excellence in Computer Applications Scholarship. The second author was supported in part by a Research Enchancement Grant from KY NSF EPSCoR (2002-4).

${ }^{1}$ Department of Mathematics, University of Tennessee at Chattanooga, 615 McCallie Avenue, Chattanooga, TN 37403-2598, USA.

2 Department of Mathematics \& Statistics, Murray State University, Faculty Hall 6C, Murray, KY 42071-3341, USA;

e-mail: maeve.mccarthy@murraystate.edu 
the disk. Let $\varphi:[a, b] \times[0, \infty) \rightarrow R$ be the angle of rotation and $s:[a, b] \rightarrow R$ be the cross-sectional area of the disk. The angle of rotation is the solution of the following initial boundary value problem

$$
\begin{aligned}
G\left(r^{3} s(r) \varphi_{r}\right)_{r}= & \rho r^{3} s(r) \varphi_{t t}, \quad r \in(a, b), t \geq 0 \\
& s(a) \varphi_{r}(a, t)-C_{1} \varphi(a, t)=0 \\
& s(b) \varphi_{r}(b, t)+C_{2} \varphi_{t t}(b, t)=0
\end{aligned}
$$

with the standard initial conditions. Here $s(r)$ is the cross-sectional area of the disk at $r$, where $a$ is the radius of the cylinder and $b$ is the radius of the disk and cylinder combined. The constants $G$ and $\rho$ are the shear modulus and the density of the material, respectively. The constant $C_{1}$ characterizes the rigidity of the attachment of the disk to the cylinder, while the constant $C_{2}$ is proportional to the mass attached to the perimeter of the disk.

We consider harmonic oscillations with the frequency $\omega$ so that $\varphi(r, t)=u(r) \mathrm{e}^{i \omega t}$. Introducing the notation $p(r)=r^{3} s(r), \lambda=\omega^{2} \rho / G, c_{1}=a^{3} C_{1}$ and $c_{2}=b^{3} C_{2} G / \rho$, the function $u(r)$ satisfies the following Sturm-Liouville problem

$$
\begin{aligned}
-\left(p u^{\prime}\right)^{\prime} & =\lambda p u, \quad a<r<b, \\
p(a) u^{\prime}(a) & =c_{1} u(a), \\
p(b) u^{\prime}(b) & =c_{2} \lambda u(b) .
\end{aligned}
$$

In the presence of a mass attached to the end of the disk, both $c_{1}$ and $c_{2}$ are positive, and the Sturm-Liouville problem has eigenparameter dependent boundary conditions. A Sturm-Liouville problem of this form is said to be regular provided that $p(r)>0$ for $r \in[a, b]$. If $p(a)=0$ or $p(b)=0$, then it is said to be singular ([1], Sect. 1.6) Physically, the natural restriction on the cross-sectional area $s(r)$ is that $s(r)>0$ for $r \in[a, b]$. Since $p(r)=r^{3} s(r)$ the Sturm-Liouville problem is regular at $a$ and $b$.

We shall refer to the Sturm-Liouville problem (4-6) as the Turbine Problem and denote by $\lambda_{1}(p)$ its least eigenvalue. In our optimal design problem, we shall maximize the first frequency of oscillation subject to the disk being of a fixed moment of inertia, $J$. Since $\lambda=\omega^{2} G / \rho$, maximizing the first frequency $\omega_{1}$ is equivalent to maximizing the least eigenvalue $\lambda_{1}(p)$.

Design Problem. We seek

$$
\sup _{p \in a d} \lambda_{1}(p)
$$

where the admissible set of functions $p(r)$ is given by

$$
a d=\left\{p: 0<p(r)<\infty, r \in[a, b], \int_{a}^{b} p(r) \mathrm{d} r=J\right\} .
$$

Note that these problems are equivalent to the minimization of the moment of inertia of the turbine subject to the constraint of a fixed first frequency or eigenvalue.

The usual approach to the problems of optimal structural design is based on the methods of Calculus of Variations. We mention only few results in this area, in particular those whose methods or applications are similar to those of this paper. The shape of the column having a given mass and length and the maximum buckling load was found in $[13,14]$. The shape of an oscillating bar having a given least eigenfrequency and the minimum mass was found in $[12,15]$. The complementary problem (design of a bar having maximum of the least eigenfrequency with the given mass) was also solved there. If we discretize an elastic structure, we reduce the problem of the optimal design to the problem of optimization for a function of several variables with constraints. This numerical approach was developed, e.g., in [15]. Note, it is more general than the analytic methods. The consideration of the shape of the tallest column was started in [8] and completed in [4]. It appeared that the 
structure of the spectrum of a certain Sturm-Liouville problem associated with the physical model is important for the applicability of the classical approach developed in [12-15]. For the more detailed review of the results in the area see, e.g. [4,5] and the references there. Our construction follows the scheme developed in [12-15]. An interesting phenomenon that did not occur in previous results is that the optimal form of the disk becomes significantly different when the parameters of the disk belong to different sets which we describe precisely.

The paper is organized as follows. In Section 1 we give some variational characterizations of the least eigenvalue of the Sturm-Liouville problem (4-6). In Section 2 we use the rearrangement techniques to prove that the least eigenvalue may be increased via decreasing rearrangements. In Section 3 we prove the existence of the optimal design. We give the necessary conditions for optimality in Section 4 . We also analyze the optimality conditions and we prove that for any given set of parameters of the model the optimal design exists and is unique. We give an explicit form of the optimal design for different sets of parameters.

\section{The SPECTRUm AND SOME VARIATIONAL CHARACTERIZATIONS}

For $p \in a d$, the Sturm-Liouville problem is regular. Thus the spectrum of $(4-6)$ consists of a discrete set of real simple eigenvalues ([1], Sect. 1.6). This implies that $\lambda_{1}(p)$, the least eigenvalue for the Turbine Problem (4-6) associated with $p$, is isolated. Three characterizations of $\lambda_{1}(p)$ will be established. The first characterization and the fact that $\lambda_{1}(p)$ is isolated will lead to our optimality conditions, while the second and third characterizations lead to our existence result.

\subsection{Characterization I of $\lambda_{1}(p)$}

The first characterization of $\lambda_{1}(p)$ is based on the standard Rayleigh quotient

$$
\lambda_{1}(p)=\inf _{u \in H^{1}(a, b)} \frac{\int_{a}^{b} p(x)\left(u^{\prime}(x)\right)^{2} \mathrm{~d} x+c_{1} u^{2}(a)}{\int_{a}^{b} p(x)(u(x))^{2} \mathrm{~d} x+c_{2} u^{2}(b)} .
$$

This characterization is used in the derivation of the optimality conditions for the design problem. Since $c_{1}, c_{2}$ and $p$ are all positive, the least eigenvalue is positive.

\subsection{Characterization II of $\lambda_{1}(p)$}

Using variation of parameters, as in [6,7], we find that if $u$ is a solution of (4-6) corresponding to $p$ then $v(x)=\sqrt{p(x)} u(x)$ satisfies

$$
v(x)=\lambda\left[\phi_{p}(x)+\left(G_{p} v\right)(x)\right]
$$

for $a<x<b$, where

$$
\begin{aligned}
\phi_{p}(x) & =\frac{\sqrt{p(x)} c_{2} u(b)}{c_{1}}\left(c_{1} \int_{a}^{x} \frac{\mathrm{d} x}{p(x)}+1\right) \\
\left(G_{p} v\right)(x) & =\int_{a}^{b} g_{p}(x, t) v(t) \mathrm{d} t \\
g_{p}(x, t) & =\sqrt{p(t)} \sqrt{p(x)}\left(\frac{1}{c_{1}}+\int_{a}^{x \wedge t} \frac{\mathrm{d} y}{p(y)}\right)
\end{aligned}
$$

and $x \wedge t=\min \{x, t\}$. If $\langle u, v\rangle$ denotes the $L^{2}(a, b)$ inner product and $\|\cdot\|$ denotes its associated norm, then this can be written as

$$
\|v\|^{2}=\lambda\left[\left\langle\phi_{p}, v\right\rangle+\left\langle G_{p} v, v\right\rangle\right] .
$$


This immediately leads to a variational characterization similar to that of Porter and Stirling ([10], Lem. 5.1)

$$
\frac{1}{\lambda_{1}(p)}=\max _{\|v\|=1}\left[\left\langle\phi_{p}, v\right\rangle+\left\langle G_{p} v, v\right\rangle\right]
$$

The maximum is attained at $v_{1}(x)=\sqrt{p(x)} u_{1}(x)$ where $u_{1}$ is the first eigenfunction of the Turbine Problem (4-6) associated with $p$. This characterization will be used later in conjunction with the Dominated Convergence theorem to establish existence of an optimal design.

\subsection{Characterization III of $\lambda_{1}(p)$}

Using $v(x)=\sqrt{p(x)} u(x)$ we find that

$$
\begin{aligned}
\left\langle\phi_{p}, v\right\rangle+\left\langle G_{p} v, v\right\rangle= & \int_{a}^{b} c_{2} u(b)\left(\int_{a}^{x} \frac{\mathrm{d} y}{p(y)}+\frac{1}{c_{1}}\right) p(x) u(x) \mathrm{d} x \\
& +\int_{a}^{b}\left[\int_{a}^{b}\left(\frac{1}{c_{1}}+\int_{a}^{x \wedge t} \frac{\mathrm{d} y}{p(y)}\right) p(t) u(t) \mathrm{d} t\right] p(x) u(x) \mathrm{d} x
\end{aligned}
$$

for $a<x<b$.

Making the substitution

$$
U(t)=\int_{a}^{t} \frac{\mathrm{d} y}{p(y)} \quad \text { and } \quad V(t)=-\int_{t}^{b} p(y) u(y) \mathrm{d} y
$$

we have

$$
\left\langle\phi_{p}, v\right\rangle+\left\langle G_{p} v, v\right\rangle=-\frac{c_{2} u(b)}{c_{1}} V(a)+\frac{1}{c_{1}} V^{2}(a)+c_{2} u(b) \int_{a}^{b} U(x) V^{\prime}(x) \mathrm{d} x+\int_{a}^{b}\left[\int_{a}^{b}(U(x \wedge t)) V^{\prime}(t) \mathrm{d} t\right] V^{\prime}(x) \mathrm{d} x .
$$

Using integration by parts, it follows that

$$
\begin{aligned}
\left\langle\phi_{p}, v\right\rangle+\left\langle G_{p} v, v\right\rangle= & -\frac{c_{2} u(b)}{c_{1}} V(a)+\frac{1}{c_{1}} V^{2}(a)+c_{2} u(b) \int_{a}^{b} U(x) V^{\prime}(x) \mathrm{d} x \\
& +\int_{a}^{b}\left[\int_{a}^{x} U(t) V^{\prime}(t) \mathrm{d} t-U(x) V(x)\right] V^{\prime}(x) \mathrm{d} x \\
= & -\frac{c_{2} u(b)}{c_{1}} V(a)+\frac{1}{c_{1}} V^{2}(a)+c_{2} u(b) \int_{a}^{b} U(x) V^{\prime}(x) \mathrm{d} x \\
& -\int_{a}^{b}\left[\int_{a}^{x} V(t) U^{\prime}(t) \mathrm{d} t\right] V^{\prime}(x) \mathrm{d} x \\
= & -\frac{c_{2} u(b)}{c_{1}} V(a)+\frac{1}{c_{1}} V^{2}(a)-c_{2} u(b) \int_{a}^{b} V(x) U^{\prime}(x) \mathrm{d} x \\
& +\int_{a}^{b} V^{2}(x) U^{\prime}(x) \mathrm{d} x .
\end{aligned}
$$


From the second characterization (14) and the definitions of $U$ and $V$, we find our third characterization

$$
\begin{aligned}
\frac{1}{\lambda_{1}(p)}= & \max _{\|u\|_{p}=1}\left[\frac{c_{2} u(b)}{c_{1}} \int_{a}^{b} p(y) u(y) \mathrm{d} y+\frac{1}{c_{1}}\left(\int_{a}^{b} p(y) u(y) \mathrm{d} y\right)^{2}\right. \\
& \left.+c_{2} u(b) \int_{a}^{b}\left(\int_{x}^{b} p(y) u(y) \mathrm{d} y\right) \frac{\mathrm{d} x}{p(x)}+\int_{a}^{b}\left(\int_{x}^{b} p(y) u(y) \mathrm{d} y\right)^{2} \frac{\mathrm{d} x}{p(x)}\right]
\end{aligned}
$$

where $\|\cdot\|_{p}$ denotes the norm associated with the $L^{2}(a, b ; p(x))$ inner product. The maximum is attained at $u_{1}$, the first eigenfunction of the Turbine Problem (4-6) associated with $p$ for which $\int_{a}^{b} u_{1}^{2} p(x) \mathrm{d} x=1$. This eigenfunction has no zeros and we assume without loss of generality that $u_{1}>0$.

This characterization will be used later in conjunction with the properties of rearrangements to establish the fact that the decreasing rearrangement of a design yields a first eigenvalue that is at least as large as the one corresponding to the original design.

\section{Application of Rearrangements}

In this section, we prove that the frequency of the disk can be increased via decreasing rearrangements. We begin by recalling a number of definitions and results from the theory of rearrangements.

Definition 2.1. The decreasing rearrangement of a nonnegative function, $f$, on $(a, b)$ is simply

$$
f^{*}(x) \equiv \sup \left\{t>0: \mu_{f}(t)>x\right\}
$$

where $\mu_{f}$ is the distribution function of $f$,

$$
\mu_{f}(t)=|\{x \in(a, b): f(x)>t\}| \quad t \geq 0 .
$$

The increasing rearrangement of $f$ is $f_{*}(x) \equiv f^{*}(b-x)$.

Remark 2.2. If $g$ and $h$ are nonnegative functions on $(a, b)$, with $g$ increasing and $h$ decreasing, then

$$
\begin{gathered}
\int_{a}^{b} f \mathrm{~d} x=\int_{a}^{b} f^{*} \mathrm{~d} x=\int_{a}^{b} f_{*} \mathrm{~d} x \\
\int_{a}^{b} f^{*} g \mathrm{~d} x \leq \int_{a}^{b} f g \mathrm{~d} x, \quad \int_{a}^{b} f_{*} h \mathrm{~d} x \leq \int_{a}^{b} f h \mathrm{~d} x .
\end{gathered}
$$

This is a special case of those established in [9] (p. 153).

By (17), if we replace a particular design $p \in a d$ by either its increasing or decreasing rearrangements $p_{*}$ or $p^{*}$ then the new design will still be in $a d$.

Remark 2.3. If $f$ is decreasing on the range of $g$ then the composition $(f \circ g)_{*}=f \circ g^{*}$. This is a special case of [3] (Th. 1).

Theorem 2.4. For $p \in$ ad

$$
\lambda_{1}(p) \leq \lambda_{1}\left(p^{*}\right)
$$


Proof. Let $u_{1}$ be the first positive eigenfunction of the Turbine Problem (4-6) associated with $p^{*}$. By the third characterization of $\lambda_{1}(p)$ given by (15)

$$
\begin{aligned}
\frac{1}{\lambda_{1}(p)} \geq & \frac{c_{2} u_{1}(b)}{c_{1}} \int_{a}^{b} p(y) u_{1}(y) \mathrm{d} y+\frac{1}{c_{1}}\left(\int_{a}^{b} p(y) u_{1}(y) \mathrm{d} y\right)^{2} \\
& +c_{2} u_{1}(b) \int_{a}^{b}\left(\int_{x}^{b} p(y) u_{1}(y) \mathrm{d} y\right) \frac{\mathrm{d} x}{p(x)}+\int_{a}^{b}\left(\int_{x}^{b} p(y) u_{1}(y) \mathrm{d} y\right)^{2} \frac{\mathrm{d} x}{p(x)}
\end{aligned}
$$

Integrating (4) with $u_{1}$ and $p^{*}$ between $x$ and $b$, and using the boundary condition (6) yields

$$
u_{1}^{\prime}(x)=\frac{\lambda}{p^{*}(x)}\left[\int_{x}^{b} p^{*} u_{1} \mathrm{~d} r+c_{2} u_{1}(b)\right] .
$$

Since $\lambda, b, c_{2}, u_{1}(x), p(x)>0$, this implies that $u_{1}$ is increasing. It follows from Remark 2.2 that $\int_{x}^{b} p u_{1} \mathrm{~d} y$ $\geq \int_{x}^{b} p^{*} u_{1} \mathrm{~d} y$ and so

$$
\begin{aligned}
\frac{1}{\lambda_{1}(p)} \geq & \frac{c_{2} u_{1}(b)}{c_{1}} \int_{a}^{b} p^{*}(y) u_{1}(y) \mathrm{d} y+\frac{1}{c_{1}}\left(\int_{a}^{b} p^{*}(y) u_{1}(y) \mathrm{d} y\right)^{2} \\
& +c_{2} u_{1}(b) \int_{a}^{b}\left(\int_{x}^{b} p^{*}(y) u_{1}(y) \mathrm{d} y\right) \frac{\mathrm{d} x}{p(x)}+\int_{a}^{b}\left(\int_{x}^{b} p^{*}(y) u_{1}(y) \mathrm{d} y\right)^{2} \frac{\mathrm{d} x}{p(x)} .
\end{aligned}
$$

Clearly, the functions $\left(\int_{x}^{b} p^{*} u_{1} \mathrm{~d} y\right)$ and $\left(\int_{x}^{b} p^{*} u_{1} \mathrm{~d} y\right)^{2}$ are nonnegative decreasing function of $x$. Another application of Remark 2.2 yields

$$
\begin{aligned}
\frac{1}{\lambda_{1}(p)} \geq & \frac{c_{2} u_{1}(b)}{c_{1}} \int_{a}^{b} p^{*}(y) u_{1}(y) \mathrm{d} y+\frac{1}{c_{1}}\left(\int_{a}^{b} p^{*}(y) u_{1}(y) \mathrm{d} y\right)^{2}+c_{2} u_{1}(b) \int_{a}^{b}\left(\int_{x}^{b} p^{*}(y) u_{1}(y) \mathrm{d} y\right)\left(\frac{1}{p(x)}\right)_{*} \mathrm{~d} x \\
& +\int_{a}^{b}\left(\int_{x}^{b} p^{*}(y) u_{1}(y) \mathrm{d} y\right)^{2}\left(\frac{1}{p(x)}\right)_{*} \mathrm{~d} x .
\end{aligned}
$$

Remark 2.3 with $f=1 / x, g=p$ implies that

$$
\begin{aligned}
\frac{1}{\lambda_{1}(p)} \geq & \frac{c_{2} u_{1}(b)}{c_{1}} \int_{a}^{b} p^{*}(y) u_{1}(y) \mathrm{d} y+\frac{1}{c_{1}}\left(\int_{a}^{b} p^{*}(y) u_{1}(y) \mathrm{d} y\right)^{2}+c_{2} u_{1}(b) \int_{a}^{b}\left(\int_{x}^{b} p^{*}(y) u_{1}(y) \mathrm{d} y\right) \frac{\mathrm{d} x}{p^{*}(x)} \\
& +\int_{a}^{b}\left(\int_{x}^{b} p^{*}(y) u_{1}(y) \mathrm{d} y\right)^{2} \frac{\mathrm{d} x}{p^{*}(x)}=\frac{1}{\lambda_{1}\left(p^{*}\right)} .
\end{aligned}
$$

\section{Existence OF AN OPtimal DESign}

In order to establish existence of an optimal design for the turbine design problem, we will use the rearrangement result of Theorem 2.4 and Helly's selection theorem ([11], p. 167), which is restated here for convenience. 
Theorem 3.1 (Helly's selection theorem). If $\left\{f_{n}\right\}_{n=1}^{\infty}$ is a sequence of nonnegative nonincreasing functions on $[a, b]$, then there exists a subsequence $\left\{f_{n_{k}}\right\}_{k=1}^{\infty}$ and a function $f$ such that

$$
f(x)=\lim _{k \rightarrow \infty} f_{n_{k}}(x)
$$

for every $x$ in $[a, b]$.

Theorem 3.2. The functional $p \mapsto \lambda_{1}(p)$ attains its maximum on

$$
a d=\left\{p: 0<p(r)<\infty, r \in[a, b], \int_{a}^{b} p(r) \mathrm{d} r=J\right\} .
$$

Proof. Recall the first characterization (8)

$$
\lambda_{1}(p)=\inf _{u \in H^{1}(a, b)} \frac{\int_{a}^{b} p(x)\left(u^{\prime}(x)\right)^{2} \mathrm{~d} x+c_{1} u^{2}(a)}{\int_{a}^{b} p(x)(u(x))^{2} \mathrm{~d} x+c_{2} u^{2}(b)} .
$$

Since $u \equiv 1$ is in $H^{1}(a, b)$,

Hence, $\lambda_{1}(p)$ is bounded above on ad.

$$
\lambda_{1}(p) \leq \frac{c_{1}}{\int_{a}^{b} p(x) \mathrm{d} x+c_{2}}=\frac{c_{1}}{J+c_{2}}
$$

Letting

$$
\hat{\lambda}=\sup _{p \in a d} \lambda_{1}(p)
$$

we see that there exists a maximizing sequence $\left\{p_{n}\right\} \subset a d$ and for which $\lambda_{1}\left(p_{n}\right) \rightarrow \hat{\lambda}$. By Remark 2.2 and Theorem 2.4, we may assume that each $p_{n}$ is nonincreasing and hence, by Helly's selection theorem, there exists a $\hat{p}$ and a subsequence (that we will not relabel) such that $p_{n} \rightarrow \hat{p}$ pointwise.

It follows by the dominated convergence theorem that

$$
\int_{a}^{x} \frac{\mathrm{d} x}{p_{n}(x)} \rightarrow \int_{a}^{x} \frac{\mathrm{d} x}{\hat{p}(x)} \quad \text { and } \quad \int_{a}^{x \wedge t} \frac{\mathrm{d} x}{p_{n}(x)} \rightarrow \int_{a}^{x \wedge t} \frac{\mathrm{d} x}{\hat{p}(x)}
$$

for each $x$ and $t$. Hence, using (10) and (12),

$$
\phi_{p_{n}}(x) \rightarrow \phi_{\hat{p}}(x) \quad \text { and } \quad g_{p_{n}}(x, t) \rightarrow g_{\hat{p}}(x, t)
$$

for each $x$ and $t$. Further application of the dominated convergence theorem yields $g_{p_{n}} \rightarrow g_{\hat{p}}$ in $L^{2}((a, b) \times(a, b))$. An argument identical to [4] (Prop. 3.3) implies that

$$
\left|\frac{1}{\lambda_{1}(p)}-\frac{1}{\lambda_{1}(q)}\right| \leq\left\|g_{p}(x, t)-g_{q}(x, t)\right\|_{L^{2}}
$$

and so $\lambda_{1}\left(p_{n}\right) \rightarrow \lambda_{1}(\hat{p})$.

However, by construction, $\lambda_{1}\left(p_{n}\right) \rightarrow \hat{\lambda}$, and so we must have $\lambda_{1}(\hat{p})=\hat{\lambda}$.

\section{NeCESSARY CONDITIONS FOR OPTIMALITY}

The first eigenvalue of the Turbine Problem (4-6) is given by the infimum of the Rayleigh quotient

$$
\lambda_{1}(p)=\inf _{u \in H^{1}(a, b)} \frac{\int_{a}^{b} p(x)\left(u^{\prime}(x)\right)^{2} \mathrm{~d} x+c_{1} u^{2}(a)}{\int_{a}^{b} p(x)(u(x))^{2} \mathrm{~d} x+c_{2} u^{2}(b)}
$$


When $p \in a d$, the first eigenvalue $\lambda_{1}(p)$ exists, is real and isolated because we have a discrete set of eigenvalues. Having already established existence of an optimal design over this class, we seek necessary conditions of optimality. We may now use standard Calculus of Variations techniques to derive an optimality condition in the form of a differential equation. The complete analysis of that differential equation along with the boundary conditions and the constraint appears to be much more complex than in $[13,15]$. We will show that the design parameter space may be split into subspaces and that a unique optimal design exists on each of them.

Consider the functional

$$
F(p)=\lambda_{1}(p)+\mu\left[\int_{a}^{b} p(x) \mathrm{d} x-J\right]
$$

where $\mu$ is a Lagrange multiplier.

At the optimum

$$
\begin{aligned}
\frac{\partial F}{\partial p} & =\frac{\left[\int_{a}^{b} p u^{2} \mathrm{~d} x+c_{2} u^{2}(b)\right] \int_{a}^{b}\left(u^{\prime}\right)^{2} \mathrm{~d} x-\left[\int_{a}^{b} p\left(u^{\prime}\right)^{2} \mathrm{~d} x+c_{1} u^{2}(a)\right] \int_{a}^{b} u^{2} \mathrm{~d} x}{\left[\int_{a}^{b} p u^{2} \mathrm{~d} x+c_{2} u^{2}(b)\right]^{2}}+\mu \int_{a}^{b} \mathrm{~d} x \\
& =\frac{\int_{a}^{b}\left(u^{\prime}\right)^{2} \mathrm{~d} x-\lambda_{1}(p) \int_{a}^{b} u^{2} \mathrm{~d} x}{\int_{a}^{b} p u^{2} \mathrm{~d} x+c_{2} u^{2}(b)}+\mu \int_{a}^{b} \mathrm{~d} x=0
\end{aligned}
$$

which yields the optimality condition

$$
\frac{\left(u^{\prime}\right)^{2}-\lambda_{1}(p) u^{2}}{\int_{a}^{b} p u^{2} \mathrm{~d} x+c_{2} u^{2}(b)}+\mu=0
$$

By continuity, and use of the boundary condition (5), the Lagrange multiplier is

$$
\mu=\frac{\lambda_{1}(p) u^{2}(a)-\left(c_{1} u(a) / p(a)\right)^{2}}{\int_{a}^{b} p u^{2} \mathrm{~d} x+c_{2} u^{2}(b)}=\frac{\left(\lambda_{1}(p) p^{2}(a)-c_{1}^{2}\right) u^{2}(a)}{p^{2}(a)\left[\int_{a}^{b} p u^{2} \mathrm{~d} x+c_{2} u^{2}(b)\right]}
$$

Hence, the optimality condition (20) can be expressed as

$$
\left(u^{\prime}\right)^{2}-\lambda_{1}(p) u^{2}+\frac{\left(\lambda_{1}(p) p^{2}(a)-c_{1}^{2}\right) u^{2}(a)}{p^{2}(a)}=0 .
$$

\subsection{Analysis of the optimality conditions}

Let $Q=\left(\lambda_{1}(p) p^{2}(a)-c_{1}^{2}\right) u^{2}(a) / p^{2}(a)$. The optimal design $p$ satisfies the boundary value problem

$$
\begin{aligned}
-\left(p u^{\prime}\right)^{\prime} & =\lambda p u, \quad a<r<b \\
p(a) u^{\prime}(a) & =c_{1} u(a), \\
p(b) u^{\prime}(b) & =c_{2} \lambda u(b)
\end{aligned}
$$

the optimality condition

$$
\left(u^{\prime}\right)^{2}-\lambda_{1} u^{2}+Q=0
$$


and the constraint

$$
\int_{a}^{b} p(r) \mathrm{d} r=J
$$

Although the explicit form of the design $p(r)$ depends on the sign of $Q$, equation (25) can be solved (for any $Q$ ) for $u(r)$, and then $p(r)$ can be determined explicitly from (22) and (24). Further, constraints on the physical parameters $J, c_{1}, c_{2}$ and $b-a$ may be found using (23) and (26), yielding the aforementioned subspaces of the design parameter space. It is convenient to consider three possible cases $Q=0, Q>0$, and $Q<0$ separately and then find the conditions on the physical parameters under which those cases occur.

4.1.1. $Q=0$

In this case

$$
u(r)=\mathrm{e}^{\sqrt{\lambda_{1}} r}, \quad p(r)=c_{2} \sqrt{\lambda_{1}} \mathrm{e}^{2 \sqrt{\lambda_{1}}(b-r)}
$$

and (23) and (26) imply that $\lambda_{1}$ must satisfy

$$
\begin{array}{r}
c_{2} \lambda_{1} \mathrm{e}^{2 \sqrt{\lambda_{1}}(b-a)}=c_{1} \\
J=\frac{c_{2}}{2}\left(\mathrm{e}^{2 \sqrt{\lambda_{1}}(b-a)}-1\right) .
\end{array}
$$

$\lambda_{1}$ can be determined uniquely from these equations

$$
\lambda_{1}=\frac{c_{1}}{2 J+c_{2}}
$$

giving a unique optimal design. We note that, by (29), this case occurs when the physical parameters $J, c_{1}, c_{2}$, $b-a$ are in the subspace described by

$$
\frac{2 J+c_{2}}{c_{2}}=\mathrm{e}^{2 \sqrt{\frac{c_{1}}{2 J+c_{2}}}(b-a)} .
$$

4.1.2. $Q>0$

In this case

$$
u(r)=\sqrt{\frac{Q}{\lambda_{1}}} \cosh \left(\sqrt{\lambda_{1}} r+\xi\right), \quad p(r)=\frac{c_{2} \sqrt{\lambda_{1}} \sinh 2\left(\sqrt{\lambda_{1}} b+\xi\right)}{2 \sinh ^{2}\left(\sqrt{\lambda_{1}} r+\xi\right)}
$$

and (23) and (26) imply that the parameters $\lambda_{1}, \xi$ must satisfy

$$
\begin{array}{r}
c_{2} \sinh 2 \beta\left(\frac{\beta-\alpha}{b-a}\right)^{2}=c_{1} \sinh 2 \alpha \\
J=\frac{c_{2} \sinh 2 \beta}{2 \tanh \alpha}-c_{2} \cosh ^{2} \beta
\end{array}
$$

where $\beta=\left(\sqrt{\lambda_{1}} b+\xi\right)$ and $\alpha=\left(\sqrt{\lambda_{1}} a+\xi\right)$. If $(32,33)$ can be solved uniquely for $\alpha$ and $\beta$ (and hence $\lambda_{1}$ and $\xi)$ then the design given by (31) is unique.

Solve (33) for $\tanh \alpha$. The use of identities implies that

$$
\tanh \alpha=\frac{c_{2} \tanh \beta}{\left(J+c_{2}\right)-J \tanh ^{2} \beta} .
$$


By definition $\beta=\tanh ^{-1}(\tanh \beta)=\frac{1}{2} \ln \left(\frac{1+\tanh \beta}{1-\tanh \beta}\right)$ with a similar expression for $\alpha$. Hence

$$
\beta-\alpha=\frac{1}{2} \ln \left(\frac{1+\tanh \beta}{1-\tanh \beta} \cdot \frac{1-\tanh \alpha}{1+\tanh \alpha}\right) .
$$

Using (34) gives

$$
\beta-\alpha=\frac{1}{2} \ln \left(\frac{c_{2}+J(1+\tanh \beta)}{c_{2}+J(1-\tanh \beta)}\right) .
$$

Use of (34) in the identities

$$
\sinh 2 \alpha=\frac{2 \tanh \alpha}{1-\tanh ^{2} \alpha}, \quad \sinh 2 \beta=\frac{2 \tanh \beta}{1-\tanh ^{2} \beta}
$$

yields

$$
\frac{\sinh 2 \alpha}{\sinh 2 \beta}=\frac{c_{2}\left(\left(J+c_{2}\right)-J \tanh ^{2} \beta\right)}{\left(\left(J+c_{2}\right)-J \tanh ^{2} \beta\right)^{2}-c_{2}^{2} \tanh ^{2} \beta} \cdot\left(1-\tanh ^{2} \beta\right) .
$$

Expand the denominator of the right hand side and re-factor

$$
\frac{\sinh 2 \alpha}{\sinh 2 \beta}=\frac{c_{2}\left(J+c_{2}-J \tanh ^{2} \beta\right)}{\left(J+c_{2}\right)^{2}-J^{2} \tanh ^{2} \beta}
$$

Using this in (32) yields

from which it follows that

$$
\left(\frac{\beta-\alpha}{b-a}\right)^{2}=\frac{c_{1} \sinh 2 \alpha}{c_{2} \sinh 2 \beta}=\frac{c_{1}\left(J+c_{2}-J \tanh ^{2} \beta\right)}{\left(J+c_{2}\right)^{2}-J^{2} \tanh ^{2} \beta}
$$

$$
\beta-\alpha=(b-a) \sqrt{\frac{c_{1}\left(J+c_{2}-J \tanh ^{2} \beta\right)}{\left(J+c_{2}\right)^{2}-J^{2} \tanh ^{2} \beta}} .
$$

Using (35) and (36), we see that the system given by $(32,33)$ has a unique solution if the function

$$
f(\beta)=\frac{1}{2} \ln \left(\frac{c_{2}+J(1+\tanh \beta)}{c_{2}+J(1-\tanh \beta)}\right)-(b-a) \sqrt{\frac{c_{1}\left(J+c_{2}-J \tanh ^{2} \beta\right)}{\left(J+c_{2}\right)^{2}-J^{2} \tanh ^{2} \beta}}
$$

has a unique zero.

We shall now show that the case $Q>0$ occurs when the physical parameters $J, c_{1}, c_{2}, b-a$ lie in the subspace given by

$$
\frac{2 J+c_{2}}{c_{2}}>\mathrm{e}^{2 \sqrt{\frac{c_{1}}{2 J+c_{2}}}(b-a)} .
$$

Substituting $u$ from (31) into (23) yields

$$
\tanh \alpha=\frac{c_{1}}{p(a) \sqrt{\lambda_{1}}}
$$


Since $Q>0$ implies that $\lambda_{1} p^{2}(a)-c_{1}^{2}>0$, and the quantities $\lambda_{1}, c_{1}, p(a)$ are all positive we must have $0<\tanh \alpha<1$. Thus $\alpha>0$. Since $\alpha<\beta$ it follows that $\beta>0$. The system $(32,33)$ has a unique solution if $f$ has a unique zero on $(0, \infty)$. Clearly

$$
f(0)=-(b-a) \sqrt{\frac{c_{1}}{J+c_{2}}}<0
$$

and

$$
\lim _{\beta \rightarrow \infty} f(\beta)=\frac{1}{2} \ln \left(\frac{c_{2}+2 J}{c_{2}}\right)-(b-a) \sqrt{\frac{c_{1}}{c_{2}+2 J}} .
$$

Since $\beta>0$ we know that $0<\tanh \beta<1$, which implies that

$$
\frac{c_{2}+2 J}{c_{2}}>\frac{c_{2}+J(1+\tanh \beta)}{c_{2}+J(1-\tanh \beta)}
$$

Use of this in (35) yields

$$
\frac{1}{2} \ln \left(\frac{c_{2}+2 J}{c_{2}}\right)>\beta-\alpha
$$

Further use of the fact that $0<\tanh \beta<1$ implies

$$
\frac{c_{1}}{2 J+c_{2}}<\frac{c_{1}}{\left(J+c_{2}\right)+J \tanh \beta}=\frac{c_{1}\left(J+c_{2}-J \tanh \beta\right)}{\left(J+c_{2}\right)^{2}-J^{2} \tanh ^{2} \beta}<\frac{c_{1}\left(J+c_{2}-J \tanh ^{2} \beta\right)}{\left(J+c_{2}\right)^{2}-J^{2} \tanh ^{2} \beta} .
$$

Use of this in (36) yields

It follows that

$$
(b-a) \sqrt{\frac{c_{1}}{2 J+c_{2}}}<\beta-\alpha .
$$

$$
\lim _{\beta \rightarrow \infty} f(\beta)>0 .
$$

Comparing (38) and (39), the case $Q>0$ occurs when the physical parameters $J, c_{1}, c_{2}, b-a$ lie in the subspace given by

$$
\frac{2 J+c_{2}}{c_{2}}>\mathrm{e}^{2 \sqrt{\frac{c_{1}}{2 J+c_{2}}}(b-a)} .
$$

It can be shown that $f^{\prime}(\beta)>0$ on $(0, \infty)$. Application of the Mean Value theorem implies that $f(\beta)$ has a unique zero on $(0, \infty)$. Thus a unique solution of $(32,33)$ exists when $Q>0$.

4.1.3. $Q<0$

In this case

$$
u(r)=\sqrt{\frac{-Q}{\lambda_{1}}} \sinh \left(\sqrt{\lambda_{1}} r+\xi\right), \quad p(r)=\frac{c_{2} \sqrt{\lambda_{1}} \sinh 2\left(\sqrt{\lambda_{1}} b+\xi\right)}{2 \cosh ^{2}\left(\sqrt{\lambda_{1}} r+\xi\right)}
$$

and (23) and (26) imply that the parameters $\lambda_{1}, \xi$ must satisfy

$$
\begin{gathered}
c_{2} \sinh 2 \beta\left(\frac{\beta-\alpha}{b-a}\right)^{2}=c_{1} \sinh 2 \alpha \\
J=\frac{c_{2} \sinh 2 \beta}{2}(\tanh \beta-\tanh \alpha)
\end{gathered}
$$


where $\beta=\left(\sqrt{\lambda_{1}} b+\xi\right)$ and $\alpha=\left(\sqrt{\lambda_{1}} a+\xi\right)$. If $(42,43)$ can be solved uniquely for $\alpha$ and $\beta$ (and hence $\lambda_{1}$ and $\xi)$ then the design given by (41) is unique. Note that this is identical to the designs obtained by Taylor [12] and Turner [15] for slightly different problems. An analysis similar to the one above shows that the case $Q<0$ occurs when the physical parameters $J, c_{1}, c_{2}, b-a$ satisfy

$$
\frac{2 J+c_{2}}{c_{2}}<\mathrm{e}^{2 \sqrt{\frac{c_{1}}{2 J+c_{2}}}(b-a)}
$$

\subsubsection{Design parameters}

If we consider the parameter space for $\left(c_{2}, J, c_{1}\right)$ then the choice of optimal design depends on whether the parameters are above, below, or on the surface

$$
\frac{2 J+c_{2}}{c_{2}}-\mathrm{e}^{2 \sqrt{\frac{c_{1}}{2 J+c_{2}}}(b-a)}=0
$$

or equivalently

$$
c_{1}=\left(\frac{1}{2(b-a)} \ln \left(\frac{2 J+c_{2}}{c_{2}}\right)\right)^{2}\left(2 J+c_{2}\right) .
$$

Thus, the choice of optimal design depends on the sign of the quantity

$$
\frac{2 J+c_{2}}{c_{2}}-\mathrm{e}^{2 \sqrt{\frac{c_{1}}{2 J+c_{2}}}(b-a)} .
$$

In particular, the sign of this quantity is the same as the $\operatorname{sign}$ of $Q$ if this is zero, then $Q=0$ and the design choice is given by (27). If this is positive, then $Q>0$ and the design choice is given by (31). If this is negative, then $Q<0$ and the design choice is given by (41). Figure 2 shows the parameter space for $b-a=10$.

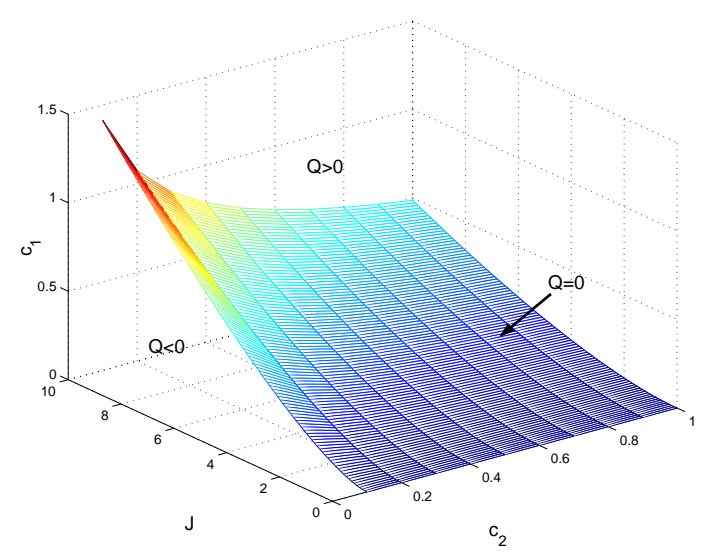

FiguRE 2. Design parameter space.

\section{Appendix}

A cylinder with an attached disk imitates different real world constructions such as a turbine with a blade. In the absence of the mass, the model also makes sense; we mention a propeller of a helicopter. The model under consideration is discussed in [2]. We give here a brief derivation of the governing equations because of the lack of an appropriate reference in English. Introduce cylindrical coordinates $\{r, \theta z\}$ where the $z$-axis 
coincides with the axis of the cylinder. Following [2], we consider the small (linear) torsional oscillations of a disk $\{r \in[a, b], \theta \in[0,2 \pi)\}$ attached to a finite cylinder $\{r \in[0, a], \theta \in[0,2 \pi), z \in[-c, c]\}$ at its middle, see Figure 1. Here $0<a<b<\infty, 0<c<\infty$, the cylinder is supposed to be fixed at its end points $z= \pm c$, and have a constant density $\rho$. Let $z=s(r):[a, b] \rightarrow R_{+}$be the variable width of the disk. A mass is attached to the perimeter of the disk and distributed uniformly along that perimeter.

We first describe a qualitative engineering analysis of the problem. The frequency of rotation in a real construction is varying in time. As a result, both torsional oscillations and oscillations in the plane of the disk appear. Generally speaking, these two types of oscillations are connected. Nevertheless we assume that connection to be small and neglect it because the forces of inertia that stipulate the oscillations sufficiently exceed the influence of two types of oscillations on each other. Hence we may ignore the radial dilatations. During the torsional oscillations of the disk its concentric circles are shifted with respect to each other. If we consider a radial line on the original surface of the disk, it becomes a curve that is characterized by the angle of twisting $\varphi=\varphi(r, t):[a, b] \times[0, \infty) \rightarrow R$. According to the (linear) Elasticity Theory, the shear $\tau(r, t)$ is proportional to the relative shift $\gamma(r, t)$ (that characterizes the variation of the original right angle between the radial lines and concentric circles). We find

$$
\gamma(r, t)=r \varphi_{r}(r, t) \text { and } \tau(r, t)=G \gamma(r, t)=\operatorname{Gr} \varphi_{r}(r, t)
$$

where $G$ is the shear modulus and the lower index denotes the partial derivative with respect to $r$. Consider an infinitely narrow ring between the limits $r$ and $r+\mathrm{d} r$ in the disk and derive an equation of motion for it. The shears create the moments with respect to the axis of the cylinder, and they are different on the boundaries. Let the moment on the interior circle be $M$ and on the exterior $M+\mathrm{d} M$; the difference $\mathrm{d} M$ stipulates acceleration of the rotation of the ring. The shear $M$ acts on the interior surface of $2 \pi r s(r)$, and hence the moment on it is $M(r, t)=2 \pi r s(r) r \tau(r, t)=2 \pi r^{2} s(r) \tau(r, t)$. We find

$$
\mathrm{d} M=2 \pi\left(r^{2} s(r) \tau(r, t)\right)_{r} \mathrm{~d} r=2 \pi G\left(r^{3} s(r, t) \varphi_{r}(r, t)\right)_{r} \mathrm{~d} r
$$

where we used the expression (A.1) for the shear. Further, the infinitely narrow ring under consideration has the moment of inertia

$$
\mathrm{d} J=2 \pi r s(r) r^{2} \rho \mathrm{d} r=2 \pi r^{3} s(r) \rho \mathrm{d} r
$$

where $\rho$ is the (constant) density of the disk. We now are in the position to write down the equation of the motion for a ring

or after obvious simplifications

$$
\mathrm{d} J \cdot \varphi_{t t}=\mathrm{d} M
$$

$$
G\left(r^{3} s(r) \varphi_{r}(r, t)\right)_{r}=\rho r^{3} s(r) \varphi_{t t}(r, t) .
$$

The equation (A.5) should be accompanied by the initial and boundary conditions. Below, we consider harmonic oscillations. Hence no initial conditions are required and only boundary conditions are to be formulated. We assume that the condition at $r=a$ has the form similar to Hooke's law

$$
s(a) \varphi_{r}(a, t)=C_{1} \varphi(a, t)
$$

where the constant $C_{1}$ characterizes the rigidity of the joining of the disk to the cylinder. Obviously $C_{1} \in(0, \infty)$. The boundary condition at $r=b$ actually represents the equation of the motion of the attached mass. The angular acceleration of that mass is stipulated by the moment at $r=b$. Proceeding as above yields

$$
0-s(b) \tau(b, t)=c_{2} \varphi_{t t}(b, t)
$$


where the constant $c_{2}$ is proportional to the attached mass. Using (A.1) finally yields

$$
-s(b) \varphi_{r}(b, t)=C_{2} \varphi_{t t}
$$

where $C_{2}$ is a constant. Obviously $C_{2} \in(0, \infty)$.

The partial differential equation (A.5) subject to the boundary conditions (A.6) and (A.7) is the subject of our further consideration.

The authors wish to acknowledge the kindness of Jon Ernstberger, Murray State University, who created the first figure.

\section{REFERENCES}

[1] L.C. Andrews, Elementary Partial Differential Equations with Boundary Value Problems. Academic Press, Orlando, Florida (1986).

[2] L. Collatz, Eigenwertaufgaben Mit Technischen Anwendungen. Akademische Verlagsgesellschaft, Geest \& Portig K.-G., Leipzig (1963) 41-42 (in German).

[3] S.J. Cox, The Two Phase Drum with the Deepest Base Note. Japan J. Ind. Appl. Math. 8 (1991) 345-355.

[4] S.J. Cox and C.M. McCarthy, The Shape of the Tallest Column. SIAM J. Math. Anal. 29 (1998) 1-8.

[5] C.L. Dym, On some recent approaches to structural optimization. J. Sounds 65 Vibration 32 (1974) 49-70.

[6] D.B. Hinton, Eigenfunction expansions for a singular eigenvalue problem with eigenparameter in the boundary condition. SIAM J. Math. Anal. 12 (1981) 572-584.

[7] D.B. Hinton, An expansion theorem for an eigenvalue problem with eigenvalue parameter in the boundary condition. Quart. J. Math. Oxford 2 (1979) 33-42.

[8] J.B. Keller and F.I. Niordson, The tallest column. J. Math. Mech. 16 (1966) 433-446.

[9] G. Polya and G. Szego, Isoperimetric Inequalites in Mathematical Physics. Princeton University Press, Princeton, NJ, Ann. Math. Stud. 27 (1951).

[10] D. Porter and D. Stirling, Integral Equations. Cambridge University Press, Cambridge, UK (1990).

[11] W. Rudin, Principles of Mathematical Analysis, 3rd Ed. McGraw-Hill, New York (1976).

[12] J.E. Taylor, Minimum mass bar for axial vibrations at specified natural frequency. AIAA J. 5 (1967) 1911-1913.

[13] J.E. Taylor, The strongest column: The energy approach. J. Appl. Mech. 34 (1967) 486-487.

[14] J.E. Taylor and C.Y. Liu, On the optimal design of columns. AIAA J. 6 (1968) 1497-1502.

[15] M.J. Turner, Design of minimum mass structures with specified natural frequencies. AIAA J. 5 (1967) 406-412. 